\title{
ARE FIT PEOPLE HEALTHY? HEALTH, EXERCISE, ACTIVE LIVING AND THE BODY IN FITNESS DISCOURSE
}

\author{
PIRKKO MARKULA \\ Department of Leisure Studies \\ University of Waikato
}

\begin{abstract}
In today's society, individuals are faced with an abundance of information detailing how to keep fit and healthy. Therefore, what advice should one follow? To answer this question, I examine what is meant by such concepts as health, physical fitness, exercise, active living and physical activity. As these concepts assume multiple meanings, for my analysis, I have chosen to examine first the scholarly research surrounding fitness and second, the material produced by the popular media. I conclude that while the research information is quite different from the popular fitness advice, both are constructed through particular social discourses. Furthermore, I demonstrate how fitness practices can become problematic. As informed consumers, we need to be aware of such problems and as educators we need to work to overcome the limitations of the current fitness practices.
\end{abstract}

\section{INTRODUCTION}

The concepts healthy lifestyle, active living and physical fitness have become very fashionable in our increasingly health conscious western world. While still a concern mainly for the middle classes, health consciousness and the fitness movement have become more visible in New Zealand in recent years. We see more people running, walking, mountain biking and roller blading than ever before. We also seem to believe that physical activity is good for our health. For example, we find that many exercisers walk or run to prevent premature heart condition. However, numerous questions abound. What kind of activity will turn us into healthy citizens? How much physical activity is needed to acquire some health benefits? And are fit people really healthy? What about all the injuries one gets while exercising or playing sport? One potential exerciser who started running, but injured his Achilles tendon and then shifted to weight training but hurt his back, concluded that it is actually healthier to stay away from physical activities. In this paper, I examine the connection between health and physical activity. I believe that understanding this connection is pertinent to any individual struggling to make sense of the abundant fitness advice saturating our newspapers, weekly magazines, and television shows. It is equally important for any fitness or health educator who prescribes physical fitness programmes to be able to assess the reliability of the fast changing fitness information.

A myriad of fitness magazines, fitness centres, fitness professionals, health researchers and health organisations push out material on physical activity and its beneficial impact on one's health. However, these sources give different guidelines on the type and amount of beneficial exercise. In this paper, I analyse 
why these discrepancies exist. Furthermore, I aim to illustrate why concepts like health, active living and exercise assume many, often contradictory, meanings in the fitness discourse. For the purpose of this paper, I will divide the fitness information sources into two categories. I will first discuss the fitness and health information targeting fitness professionals and fitness educators. This information consists of fitness and health related research and professional magazines that apply research results to the fitness industry. The first part of my paper focuses on the research based fitness information. I will examine the definition of health and the understanding of healthy fitness practices in this literature. In addition, I will outline possible problems arising from this approach to physical activity. In the second part of my paper, I will look at the information targeting the general public. We are bombarded with fitness messages from the popular media. New fitness related magazines have hit the market; television broadcasts fitness documentaries; numerous advertisements and infomercials sell exercise equipment and dieting programmes. In this part of my paper, I will examine how the popular media promotes fitness and how this approach to physical activity can be problematic. I will conclude with suggestions for overcoming the limitations to these two approaches to physical activity.

\section{HEALTH-RELATED FITNESS RESEARCH: PHYSICAL FITNESS OR PHYSICAL ACTIVITY?}

Fitness promoters in general assume that physical activity is beneficial for health. However, activity recommendations are anchored in the definition of health created by the researchers who establish the present exercise guidelines. The definition of health underlining the research on physical activity has varied over time. Initially, health was equated with the absence of illness. As long as one did not have a diagnosed illness, s/he was considered healthy. Following this understanding of health, physical activity was considered beneficial because it had the capacity to prevent illness. However, this definition was problematic in two senses. First, many human conditions that affect our capacity to function effectively were not recognised as a disease. For example, bad diet, due to poverty or fashionable junk food, could cause malfunctioning, but not necessarily a full-blown illness. Moreover, stressful lifestyle could effect one's psychological wellbeing, but many of these psychosomatic conditions would not be diagnosed as illnesses. Second, as medicine has developed effective means to control such diseases as diabetes and asthma, many of the sufferers have started to live "normal" lives. However, according to this definition of health, these people would never be considered healthy because of their chronic illness. It is clear that a more comprehensive definition of health was needed.

Pertinent for discussion in this paper is the definition of health commonly accepted by professionals working in the area of physical activity and fitness. This definition is based on the World Health Organisation's official understanding of health as a state of complete physical, mental, and social well-being rather than merely the absence of illness (Crawford, 1980). The following definition was officially stipulated in a consensus meeting of 100 international experts on physical activity and health in 1992. According to their report, heath is: 


\begin{abstract}
A human condition with physical, social and psychological dimensions, each characterised on a continuum with positive and negative poles. Positive health is associated with a capacity to enjoy life and to withstand challenges; it is not merely the absence of disease. Negative health is associated with morbidity, and in the extreme, with premature mortality (Shephard, 1995, p. 289).
\end{abstract}

This definition moves away from understanding physical activity merely as a means for preventing disease. Accordingly, exercise guidelines should be designed to increase our overall wellbeing and lifelong enjoyment of life. Still, the recommendations for the amount of exercise we should be doing differ. The differences between the recommendations are due to the difference between the terms physical fitness and physical activity. Although physical fitness and physical activity are often used as synonyms in every day conversation, they are fundamentally different concepts for the experts designing fitness programmes. Consequently, to select a suitable fitness programme, it is crucial for any fitness consumer to understand this difference.

Many researchers distinguish between physical activity and physical fitness. Physical activity is defined as bodily movement by the skeletal muscles. This movement results in energy expenditure that is higher than in the resting stage. Although physical activity is generally agreed to have a beneficial influence on health, being physically active is not necessarily a predictor of health. This notion leads into the distinction between physical activity and physical fitness. To perform physical activities we need some degree of physical fitness. The better our physical fitness, the better our ability to perform movements. Further, the better our physical fitness, the better our health is predicted to be. Researchers have further broken down physical fitness into skill-related and health-related fitness. Skill-related or motor fitness is performance oriented and often related to improvement in high performance physical activities like sport or dance.

For the purpose of this paper, I focus on health-related fitness as this concept is oriented towards on improvement in health rather than high performance. Although the understanding of health related fitness slightly differs between different researchers (Malina, 1994; Shephard, 1995), it has commonly been seen to consist of four components: cardio-vascular fitness, flexibility, muscle strength and endurance, and body composition. These components are objectively measurable thus, researchers have been able to test exactly how much exercise will result in improvement of each component and thus, improvement in general physical fitness. Increased physical fitness has a strong link with illness prevention. There is convincing evidence that improved physical fitness (as a result of regular exercise) can prevent coronary heart disease and related hypertension (blood pressure), type II diabetes, some types of cancer, osteoporosis, depression and anxiety. There is suggestive evidence of benefit from exercise for peripheral vascular disease, mild obesity, chronic arthritis and chronic lung disease (Blair, Brill \& Barlow, 1994; Haskell, 1994; Kravitz \& Robergs, 1993; Paffenberger, Hyde, Wing, Lee \& Kambert, 1994; Pollock, Feigenbaum \& Brechue, 1995; Shephard, 1995). Each of these conditions benefit from the improvement of one or more of the components of physical fitness. 
Exercise is a type of physical activity that has been designed specifically to improve each component of physical fitness. Exercise, thus, is repetitive, regular and consists of increasing amounts of physical activity. Exercise duration, frequency and intensity determine the exact amount of exercise individuals need for improved physical fitness. Most common exercise prescriptions are based on the need for improved physical fitness. For example, the American College of Sports Medicine (ACSM) originally published guidelines for recommended exercise doses in 1978. While it has revised its guidelines several times, the recommended exercise amount according to the ACSM (1990) guidelines is 3-5 times/week (frequency), 20-40 minutes (duration) at the level of $65-90 \%$ of maximum heart rate (intensity) (eg., Blair, Brill \& Barlow, 1994; Pollock, Feigenbaum \& Brechue, 1995). This recommendation translates into a weekly exercise routine consisting of two major parts: a) three times, 30 minutes of activity like aerobics, swimming, cycling or running to improve cardio-vascular functioning and body composition; and b) resistance training twice a week to improve muscle strength and endurance and bone density. Regular exercise of this dose will create a training effect: one's physical fitness will improve. In addition, the exerciser needs to regularly monitor the exercise amount. As the body gets used to the current exercise load, beneficial training effect (improvement) will no longer occur. Therefore, one needs to regularly "overload:" to increase the amount of exercise to continue gaining fitness benefits. Because regular exercise has been proven to results in significant health benefits in terms of preventing or treating illnesses, some researchers strongly believe that the ACSM exercise guidelines are appropriate for the majority of the general population (Pollock, Feigenbaum \& Brechue, 1995).

Despite all the beneficial outcomes of exercise, the number of sedentary people in the population remains high. For example, in Canada only $10-15 \%$ of adults participate in regular physical activity, while $40 \%$ are completely sedentary (Bouchard, 1994). In the United States, 22\% of adults participate in regular physical activity, but $25 \%$ are not active at all. More than $60 \%$ of adults do not achieve the recommended amount of exercise (Kravitz \& Robergs, 1993; A Report of the Surgeon General, 1996). In New Zealand, while nearly half of the adult population engages in at least some form of vigorous physical activity once a week, the other half is not physically active at all (56\%). Furthermore, the number of sedentary New Zealanders increases with age (Ministry of Health, 1995).

Why aren't more people exercising? Most people are aware of the positive impact of exercise on their health. However, it has been found that the amount of exercise required to improve physical fitness has resulted in poor adherence rates. The drop out rates for such vigorous exercise programmes are typically $50 \%$ or more after six months (Dishman, 1982; Blair, Brill \& Barlow, 1994). This has led researchers to question whether exercise that results in improved physical fitness is needed in order to gain health benefits (Haskell, 1995). Consequently, some researchers have begun to promote physical activity for health instead of exercise prescription for physical fitness (Pollock, Feigenbaum \& Brechue, 1995).

New physical activity guidelines have been developed to emphasise the role of physical activity. For example, the American Heart Association (AHA) and the Centers for Disease Control and Prevention (CDC) together with ACSM have established new standards for physical activity in 1995. Also, the well publicised 
report by the American Surgeon General (1996) regarding physical activity and health focuses on recommending physical activity instead of exercise. There are two basic differences between these new guidelines and the earlier ACSM (1990) guidelines. First, the new guidelines recommend moderate intensity activities instead of exercise at $60-90 \%$ of one's maximum heart rate. The intensity of physical activity is no longer established as a percentage of maximum heart rate. Instead, a list of recommended moderate intensity activities is provided. One example of moderate intensity activity is brisk walking. Second, some of these new guidelines advise performing multiple short bouts of activity instead of the earlier recommendation of 20-30 minutes of continuous exercise. Thus, the recommendation proposes that:

... performing a series of short bouts of moderate-intensity activity-each lasting up to 10 minutes, with the total duration being at least 30 minutes per day--will provide significant health benefits. The recommendations assume it is the total amount of activity performed at moderate intensity that is most important, not the length of a single exercise bout (Haskell, 1995, p. 46).

These new physical activity guidelines ground their recommendation on "active living" for a healthy lifestyle instead of exercise for improved physical fitness. Although some researchers are sceptical about the meaning and usefulness of the concept, active living, others have found it a helpful tool to incorporate more natural forms of physical activity into people's lives. According to Edwards (1994), active living differs from the previous understanding of fitness because it places an emphasis on daily activities, including the energy expended in work and chores. For example, during a workday, one can walk 10 minutes from one building to another and do that three times. This way a daily exercise dose is incorporated in one's daily activities. Moreover, The Surgeon General's Report recommends moderate intensity activities including washing and waxing a car, cleaning windows and gardening. These new active living guidelines, however, have not passed without controversy. In particular, some exercise scientists have criticised the recommendation of lowering the intensity of physical activity.

These researchers argue that we should adhere to the ACSM (1990) guidelines because these exercise intensity levels have been scientifically proven to improve physical fitness and thus, guarantee health benefits. They ground their arguments on the fact that it is possible to conduct rigorous scientific research regarding the components of physical fitness as they are clearly measurable. Consequently, the experiments can determine the exact effects of exercise intensity through controlled laboratory testing. For example, Pollock, Feigenbaum and Brechue (1995) describe how the effectiveness of exercise programmes (and therefore, their positive impact on health) is evaluated according to improvements in such measurable descriptors of physical fitness as VO2max (maximal oxygen intake), body composition (body weight, fat, and muscle mass), and muscular strength and endurance.

Clearly, it is more difficult to measure the exact impact of every day physical activity on one's health, as many of the benefits are subjectively determined or even unknown. However, some experts are calling for more inclusive methods of 
measuring the impacts of daily physical activity (Haskell, 1994). They indicate that a lack of research methods is no excuse to exclude moderate physical activity from the exercise guidelines. Other researchers point out flaws on the measurements used in the experiments determining the required exercise intensity for health benefits. For example, Haskell (1995) discusses the distinction between relative intensity and absolute intensity which the researchers have ignored while determining the exercise guidelines. Intensity can be established relative to each person's capacity for a specific type of exercise. For example, walking at a moderate pace might be a high intensity activity for an elderly woman, but a very light intensity activity for a male elite athlete. Absolute intensity measures do not take individual abilities into account. Rather, intensity is expressed in absolute terms like walking for 30 minutes at $70 \%$ maximum heart rate. Haskell (1995) argues further that all research demonstrating the benefits of exercise on decreased cardiovascular or all-cause mortality rates has been based on absolute intensity. Most exercise guidelines, like the new CDC/ACSM guidelines, express their recommendations in terms of absolute intensity (Haskell, 1995). The active living approach has been designed to overcome the limitations of the guidelines based on absolute intensity, but is the concept of active living the answer to decreased inactivity levels?

\section{EXERCISE AS ILLNESS PREVENTION: A PROBLEMATIC APPROACH?}

The active living based guidelines have made physical activity more convenient for people by lowering the intensity level and amount of exercise to a more realistic level. However, exercise (ACSM guidelines) and physical activity (active living) guidelines share one thing in common: both are based on the premise that physical activity prevents illness. Despite the adopted broader definition of health, physical activity and physical fitness are still pushed forward mainly because they can control chronic illnesses that require expensive and continuous health care. For example, Pollock, Feigenbaum and Brechue (1995) explicitly state that "physical activity has been shown to substantially reduce the risk of several controllable and degenerative diseases and to improve both the quality of life and longevity" (p. 320). They cite extensive literature to demonstrate how physical activity can reduce the risk of morbidity and mortality of several serious diseases. They equate an illness free existence with living a high quality life, as they do not point out any other benefits for physical activity. It is noteworthy that the guidelines promoting moderate intensity physical activity are also pushed forward by the Centers of Disease Control and Prevention (CDC) and the American Heart Association (AHA). These organisations are particularly interested in primary and secondary prevention of such illnesses as coronary heart disease, hypertension and diabetes. The link with prevention of cardio-vascular disease (CAD) and physical activity is obvious. For example, the AHA identified physical inactivity as a risk factor for the development of CAD, joining hypertension and lipid abnormalities (Pollock, Feigenbaum \& Brechue, 1995). In addition, they estimated that such illnesses contribute substantially to the $\$ 900$ billion spent annually on health care in the US. Some research identifies CAD as probably the biggest cost for the American health care system. Logically, the most often publicly cited reason for promoting physical activity is the economics of 
health care. Sparkes (1991) and Featherstone (1991) note that health-related physical activity programmes often justify their substantial governmental funding based on the calculated potential savings in public health costs. For Sparkes (1991), however, the connection between health related fitness and the prevention of cardio-vascular disease is not as clear as it may seem.

Sparkes (1991) observes that the emphasis on preventing CAD has led the health-related fitness movement to emphasise certain aspects of health while ignoring others. Prevention of CAD provides the central justification for most health-related fitness programmes, including physical education programmes in schools. While this justification seems more than adequate--after all, CAD has been portrayed as the modern killer in western society--Sparkes questions the taken-for-granted manner we believe in the epidemiological increase of CAD and consequently, the role of physical activity in its prevention. He argues that like any phenomenon in society, disease is also socially constructed. Relying on research by Bartley (1985), Sparkes illustrates how the increase in deaths due to CAD can be attributed to the changes in death certification practices. Bartley demonstrates that deaths that earlier might have been coded simply as old age, bronchitis, or arteriosclerosis are now coded as heart disease. Furthermore, many people die of "heart attacks" because they have had a pre-existing chronic illness, they are unemployed or have unhealthy working conditions. This suggests that heart disease is a consequence of a prior condition, not the primary cause of death. This leads Bartley to ask: "Why...has it been the (largely unobserved, imputed) state of the coronary arteries which has been written into the orthodoxy of health education and the popular consciousness, rather than the unemployment and chronic illness?" (p. 307). Bartley suggests that CAD serves as a convenient rationale for many puzzling incidents. For example, an emphasis on CAD has been devised to explain an unfavourable change of health in certain populations (eg. working class men) and sudden deaths in suspicious and medicolegally ambiguous cases. Therefore, medicine can mask societal problems and inequalities of health care by describing a myriad of conditions as heart disease. As a result, Sparkes (1991) continues, the medical profession has identified circulatory and heart disease as a major contemporary health problem and an active lifestyle as a key preventive measure. Subsequently, it becomes logical to ignore the societal causes of sudden deaths and attribute the prevention of CAD upon the individual's shoulders.

Maintaining a physically active lifestyle, for many, is a personal choice: it is up to the individual to adopt proper exercise practices and be responsible for one's health. Through such beliefs, Sparkes (1991) demonstrates, health-related fitness is defined within a framework of individualism. As one of the most prevailing ideologies in western culture, individualism maintains that the "individual is fully responsible for his or her own actions and life situation" (Sparkes, 1991, p. 213). However, categorising health-related fitness as an individual's responsibility can attribute social problems to individual people. For example, Ingham (1985) criticises campaigns that encourage us to take up jogging or cycling but conveniently ignore the fact that most people have to exercise in very polluted urban environments. These campaigns, Ingham continues, also ignore the fact the many people cannot afford exercise equipment, exercise time or health club memberships. Sparkes (1991) elaborates that while individuals can take some 
responsibility for their health, "many groups in our society have less freedom of choice than others to pursue a healthy and active lifestyle and that their lives and experiences are structured in such a way as to increase their chances of suffering illness" (p. 214). Therefore, social causes for ill health can be ignored, when we assume that individuals are solely responsible for their freely made health choices.

According to Crawford (1980), individualism is further problematic because of its moral connotation. As it is our voluntary choice to be "good," healthy citizens, by falling ill we fail to make the required commitment to health. Sick individuals are a burden to others in society. As Crawford (1980) describes: "the failure to maintain health is ascribed to some kind of unwillingness to be well or an unconscious desire to be sick, or simply a failure of will" (p. 379). Therefore, individuals are blamed for catching an illness; they somehow deserve to be ill as they have neglected their health. For example, they have been too lazy to maintain a regular exercise programme. Therefore, the victims of disease are blamed for malpracticing their health, burdening the already crowded healthcare institutions and increasing the public spending on health.

In conclusion, exercise and physical activity guidelines are designed primarily to prevent illness. However, this rather singular understanding of health as absence of illness can be problematic. It can mask the social causes and other factors contributing to ill health. This can lead to victim blaming and moral judgements of sick individuals. In addition, seeing physical activity as a way to limit spending on public health can attribute the responsibility of health and physical fitness maintenance solely to the individual. However, many people do not have the freedom to opt for a "healthier" lifestyle. Obviously, health based exercise and physical activity guidelines are cemented to social agendas invisible at first glance. The debates about the premise of these guidelines appear mostly in the professional research. Most "ordinary" exercisers derive their fitness information from more accessible sources. This leads me to the second part of my paper where I will examine the fitness information targeting the general public. As this information commonly reaches us through magazines and television, I will now examine how fitness is presented in the popular media.

\section{FITNESS IN POPULAR MEDIA: HEALTH AND BODY}

Fitness information has become a regular feature in New Zealand's popular media. Magazines recommend weight training for a tauter body shape, infomercials sell abdominizers to tighten tummies, weight loss programmes offer advise for a leaner, "swimsuit" ready body shape. It seems that the media approach fitness quite differently from the researchers of physical fitness. To clearly demonstrate the type of information they provide, I will use one magazine, New Zealand Fitness (NZF), as my primary example of a "typical" popular source for physical activity advice. NZF is a New Zealand published fitness glossy. It is widely available in bookshops, news agents and supermarkets, but it is also part of Les Mills World of Fitness Gym membership and is thus, produced by the Les Mills Gym. Les Mills is New Zealand's largest health club chain and is in many ways a "trend setting" commercial fitness centre in this country. As a promoter for Les Mills Gym, what kind of information does NZF contain? 
Typically, NZF cover pictures a well known New Zealander most often from the entertainment industry. Recently, Lana Coc-Kroft, Neil Waka, Rachel Hunter, Arlene Thomas, Lynette Forday, Jayne Mitchell and Rene Naufahu have posed for the cover. Inside the magazine these beautiful people tell how they keep their bodies fit. In addition to exercise tips from the famous people, the magazine offers weight loss, nutrition and weight training advice; information on the latest exercise equipment or latest trends in bodybuilding and competitive aerobics. In addition to the fitness information, NZF also regularly compiles displays of "the best bodies" in New Zealand. While many of these "best bodies" come from sport or fitness industry, several photographs present them in seductive or revealing poses instead of in action in their sport. Lately, NZF has also added "photo essays" celebrating the beauty of nude, sculpted bodies. Obviously, there is a strong emphasis on reshaping the body. In the pages of NZF, exercise and physical fitness have become connected to bodily appearance. As Les Mills Gym links its fitness mission strongly to altered body shape through the visible advertising slogan "Become Some Body," it is no surprise that in NZF the beautiful bodies are a central feature.

Commonly, the popular fitness media tend to provide exercise and nutrition information in relation to altered appearance. Unlike the professional literature on physical activity, the popular media seldom justifies fitness with its ability to prevent illness. Even the television documentary "Fighting Fat, Fighting Fit" viewed on TV1 in 1996, offered mixed messages about the importance of slimness and exercise as a means to a thinner figure. Although diseases connected with obesity were mentioned, there was a strong message about the necessity to change from fat to thin for the sake of more acceptable appearance. It is clear that in the popular consciousness the boundaries between health, beauty and fitness are blurred. In our culture health ideals and physical beauty ideals have become congruent. As White, Young and Gillett (1995) observe, health has increasingly become inscribed on the body. To explain this body centredness, Featherstone (1991) locates present body practices within the context of "consumer culture." Consumer culture is characterised through several features. In what follows, I will explain each characteristic through examples from New Zealand fitness discourse.

In consumer culture, the bodily appearance and "the look" have become increasingly important. According to Featherstone (1991) the emphasis on body suggests two categories. The inner body refers to the concern with health and optimum functioning of the body. "The outer body refers to appearance as well as the movement and control of the body within social space" (p. 171). Particularly pertinent to understanding the relationship between health and bodily appearance in popular culture is Featherstone's notion that in consumer culture "the inner and the outer body become conjoined: the prime purpose of the maintenance of the inner body becomes the enhancement of the appearance of the outer body" ( $p$. 171). Therefore, health practices in consumer culture are designed to improve one's appearance, yet simultaneously they are justified through health reasons. Again, the television programme "Fighting Fat, Fighting Fit" can serve as an example. It combined illness prevention (inner body) with social reasons (appearance related to outer body) to convince people to lose weight. Women, in particular, confront social pressures to look thin and many women use a changed 
appearance as a way to motivate them to do cardio-vascular exercise. In this way cardio-vascular exercise becomes primarily a practice for altered slimmer appearance.

To maintain the proper bodily "look" in consumer culture one requires numerous products and services. When individuals combat bodily deterioration and decay, they need to consume. According to Featherstone (1991) mass consumption characterises late capitalism and body maintenance provides an expanding market for the sale of body products. These products, of course, are marketed effectively through advertisements. It is no wonder, then, that television is filled with infomercials selling exercise equipment "that really works" or dieting programmes that "guarantee you to lose pounds."

Featherstone (1991) adds that the messages of body maintenance are combined with "the notion that the body is a vehicle of pleasure and selfexpression. Images of the body beautiful, openly sexual and associated with hedonism, leisure and display, emphasises the importance of appearance and the 'look"' (p. 170). To enjoy such "heightened pleasures" the individual must look good and the look, of course, is obtained through a strict exercise regimen. Moreover, Featherstone observes, that dietary control and exercise are claimed to enhance sexual prowess. One indication of such an effect are neologisms like "sexercise" and "exersex" (p. 182). NZF offers occasional tips on how exercise can energise one's sex life. For example, one article titled "Sexercise" explains how exercise helps to provide stamina for maximal sexual pleasure. Exercise, the article continues, creates a more beautiful body and thus, more confidence in sex life. NZF also provides information on impotence and on substances to boost men's sexual drive.

Within consumer culture, then, the body and the bodily pleasures are openly celebrated (Featherstone, 1991). Fitness lends itself well to the jubilation of the sexual body. The NZF nude series and "the best body" collages exemplify the connection between beauty, fitness, pleasure and sexuality. The models pose in full body shots that are openly sexual and their facial expressions signify sexual invitation. Featherstone (1991) identifies clothing as an important part of consumer culture where an unashamed display of the human body is permitted and clothing is specifically designed to applaud the beautiful body.

In this body obsessed society, however, not just any body is admirable. Featherstone (1991) observes that consumer culture longs to preserve youth and beauty is closely connected to youthfulness. In the pages of NZF we see only young people. Some celebrities also reveal their exercise secrets that help them to retain their youthful looks. For example, Neil Waka details his exercise programme for the best body of his life by age 40 and Jayne Mitchell tells how she has achieved a better look in her thirties than in her twenties. In addition to youthfulness, White, Young and Gillett (1995) elaborate, the western beauty ideal emphasises both slimness and muscularity, but in different ways for men and women.

In NZF, men are often pictured weight training accompanied by advise for increasing one's muscle size. For example, men are advised how to build "megamuscles" or "monster muscles." Special feature articles are devoted to abdominal training. One article focusing on awesome abs advises how to build washboard abdominals. Therefore, the male ideal seems to centre around 
muscularity and particularly, abdominal and upper body muscularity. Often the photographs of the males include only upper body shots. For example, all the covers picturing male models display only the models' bare upper bodies. White and Gillett (1994) point out that such muscularity is of little use for today's male as many men's work involves little physical strength. However, they add, muscles have increasingly important social value for men in today's changing society. They argue that today, women have entered many institutions previously dominated by men. Consequently, the traditional notions of masculine power have been challenged and in this turmoil, many men have lost a clear sense of what it means to be a male. Building muscles helps some men to re-define themselves as males in an environment where other opportunities for "traditional" masculinity have become increasingly scarce. These researchers claim that muscles are markers of embodied masculine power: muscles are used as a sign of dominance, control, authority, physical strength, and power. Obviously, muscularity encourages the dominant masculinity and simultaneously stresses the clear and natural, biological difference between men and women. Women's fit body in NZF, consequently, is represented quite differently from men's fit body.

While muscles are the defining feature of an admirable male body, an admirable women's body is primarily thin. All the women pictured in NZF are slim. To accentuate thinness as a crucial characteristic of a beautiful woman, the magazine uses fashion models as advisers of exercise and good nutrition. A sleek female figure seems to be an important selling point for the magazine, as even articles that do not directly refer to women or weight loss are accompanied with illustrations of thin, scantily clad women. In addition to thinness, NZF emphasises muscle tone as an important part of the female body. Unlike men's training advise which focuses on muscle size, women's training celebrates grace and style including advice on how to look "feminine" despite weight training. For example, one cover model, Rhonda McHardy, explains that she has toned down her upper body to create a smoother look. Similarly, she now runs to obtain supple, but not bulky thighs. As NZF favours the "feminine" toned look, it also supports a new fitness version of women's bodybuilding called body shaping. This competitive sport targets women who enjoy weight training but not the defined musculature of a bodybuilder. NZF often displays the healthy, toned and athletic champion shapers. One photoessay celebrates such fit and feminine bodies in still, seductive poses strongly emphasising the legs and the rear ends of the models. In general, NZF prefers to photograph female models posing passively instead of capturing them in the midst of physical activity. While the text might focus on exercise and diet routines, and some models might carry weights, wear running shoes or workout clothes, the pictures exhibit their toned bodies in immobile positions. Some photographs, like displays of female celebrities in underwear (Lana Coc-Kroft), in bikinis (Suzy Aiken) or naked (Jayne Mitchell), seem entirely unrelated to fitness issues.

Evidently, the women smiling at us from the pages of NZF all represent a singular body type: young, thin and toned. Several feminist researchers label this image as the ideal female body in today's society. In addition, this ideal is very narrowly defined: not too old, not too flabby, not too muscular. They observe other curiosities about this ideal image. It seems too resemble a body of an adolescent female or a young male--narrow hips, lanky body, sinewy muscles-- 
rather than a mature woman with rounded hips, thighs, breasts and arms (eg., Bartky, 1988; Bordo, 1993; Chernin, 1981; Markula, 1995; Spitzack, 1990). Despite the boyish look, the women are often pictured smiling in still poses, emphasising the gracefulness and sexual allure of their toned bodies. To explain this paradox, feminist researchers connect such a presentation to our patriarchal society. In this society, women's (and men's) bodies are defined according to the ideology of masculinity. This ideology bipolarises femininity and masculinity. This strict distinction is based on an understanding of natural biological differences between the female body and the male body. The biological traits connected to males (muscularity, strength, height, large body size) are seen as superior to the attributes connected to females (thinness, physical weakness, litheness, small body size). Therefore, patriarchal definitions assign women as servants for males and objects for the male gaze. Physical activity serves as an arena for the symbolic representation of males as active subjects and females as passive objects. NZF exhibits variations of the traditional stereotypes of masculinity and femininity. This manifests in the presentation of females as passive sexual objects for the male gaze (passive, near nude, graceful, whole body photographs) and males as strong and active subjects (muscular bodies sweating in the effort of lifting massive weights). NZF's focus on figure shaping instead of women's bodybuilding can be understood in a similar light.

If we look at gender relations through the lens of the ideology of masculinity, muscular, bodybuilding women are disconcerting. They threaten to disrupt the equation of men with strength and women with weakness (Holmlund, 1989). The toned bodyshapers, instead, satisfy the feminine requirements perfectly. Their bodies, unlike the "unnaturally" big bodybuilders' bodies, are graceful and sexy. Conversely, the male bodybuilders with huge bulging muscles epitomise the strong masculine image NZF regularly features through stories on male bodybuilding.

In consumer culture, the body ideals have developed into extremes: extreme thinness characterises the female beauty and extreme muscularity characterises the male ideal. As these ideals are closely connected to gender differences, many people seek to create such bodies to realise themselves as truly feminine or truly masculine. Foucault (Martin, Gutman \& Hutton, 1988) labels such a search for one's true identity as a "truth game." Frank (1991) illustrates how diet can be a truth game. The saying "imprisoned in every fat man a thin one is wildly signalling to be let out" (p. 57) implies that the thin man inside is the true self and the diet a disciplinary regime to free him. In today's consumer culture, where thinness is the defining characteristic of the female body ideal, many women seek their true selves through a combination of diet and exercise. Many women believe that their lives will be drastically altered once they lose weight. The fitness industry utilises this notion effectively when they advertise exercise programmes as slimming programmes. For example, Contours, a women's only gym, often includes customer comments on their advertisements to attract more clients. The successful exercisers attribute their weight loss to joining Contours and explain how they now feel terrific, like a new person. One women rejoices: "I've tried every diet known to women. Nothing's worked as well as my Contours membership for losing weight and toning up my body...I'm a new woman." If women aim to free their true thin selves, men's search for masculinity has a 
different focus. White and Gillett (1994) looked at how bodybuilding advertising appeals to men's hidden desire for muscular masculinity. These advertisements persuasively contrast the skinny weaklings with the powerful, dominant and muscular bodybuilders to emphasise the desired direction for the body transformation. Although these body ideals are believed to derive from natural biological body types, most men and women find them difficult to obtain.

\section{EXERCISE FOR IMPROVED APPEARANCE: A PROBLEMATIC APPROACH?}

It is obvious that most people do not naturally possess the ideal body shape and therefore, we have magazines like NZF to advise us on body reshaping. However, achieving the ideal requires hard work. Even with the strictest "body work" regimen most men and women will never, despite all their efforts, look like the magazine models. Most of us do not have the biological make-up for the ideal body. For example, it has been estimated that $5 \%$ of women are born with the right genes for the present slim ideal. Moreover, it is difficult to find the time required to effectively reshape one's body. This becomes increasingly problematic with aging, which often exacerbates the "problems" we have with our bodies. As we become older we tend to store fat more easily (women especially on the "problematic" thighs, underarms and hips); our metabolism slows down; and our muscle mass tends to decrease. Obviously, we are doomed to fail our body shaping mission. This consistent failure makes the emphasis on a bodily look a problematic philosophy for the fitness industry.

As our reshaping efforts become frustrating, we become increasingly selfcritical of our bodies. The discrepancy between the ideal body and our own defective body has resulted in great body dissatisfaction, particularly among women. Women especially are vulnerable to body dissatisfaction because women's identity is closely attached to appearance, whereas men's identity is still defined largely based on their achievements--education, job, wealth or athletic success--in life (Arveda Kissling, 1991). According to Spitzack (1990) the attractive female body has come to signify a controlled mind and healthy self-confidence and these are the characteristics of a successful woman. NZF clearly focuses on displaying the bodies of successful women on its pages instead of their achievements. While their success in work or sport might be mentioned in the text, the majority of the articles focus on body maintenance. One such example is an article about Labour leader Helen Clark who reveals her "keep slim tips" for readers instead of tips for improved access to physical activity for women. To further complicate their body mission, women's body ideal is more narrowly defined than men's. Women are exposed to more detailed body scrutiny as women's fashion generally is tighter fitting and more revealing than men's fashion. The photographs in NZF definitely allow a close scrutiny of the female models' entire bodies in revealing clothing. Women themselves learn to monitor any changes in their bodies, particularly in "the problem spots" (hips, thighs, abdominals, underarms) and consequently, spend considerable time and energy worrying about their body shapes (Markula, 1995; Spitzack, 1990). Some researchers claim that men are increasingly affected by a similar body logic as women. Many men spend time reshaping their bodies toward the muscular ideal. 
Their desperate attempts to build a better body, can lead to very rigid, overly disciplined and harmful exercise practices (White, Young \& Gillett, 1995).

The extreme body ideals--the very thin women's ideal and the very muscular men's ideal--have contributed to an increasing dissatisfaction with one's body shape and in the worst case this dissatisfaction can expand into a body-related disorder. Women's extreme fear of fat has led to clinical eating disorders such as anorexia nervosa and bulimia nervosa. Research reports a dramatic escalation of diagnosed cases of anorexia and bulimia in the 1980s and 1990s (Bordo, 1993) and approximately $90 \%$ of these cases are women (Peters, 1995). It has been estimated that $1-10 \%$ of western women suffer from an eating disorder (Bray, 1994; Wolf, 1990). The recovery rate from an eating disorder is grim: $5-15 \%$ die in treatment and about $50 \%$ never recover completely. Both anorexia and bulimia are now recognised as clinical, psychopathological disorders with established medical and psychiatrical diagnoses and treatments. However, some researchers reveal another category of body-related disorders which they label as sub-clinical disorders since there is no official pathology for these conditions (Bray, 1994). Subclinical disorders include obsessive behaviours such obsessive exercise to reduce body fat, compulsive physical training for no obvious reason and compulsive eating with out-of-control eating binges followed by excessive exercise.

Dissatisfaction with one's body can also lead to so called body image distortion. Although Body Image Distortion Syndrome (BIDS) has long been characterised as one of the symptoms for eating disorders (Bordo, 1993), it now appears as a body related disorder particularly in women's magazines. Affected by BIDS the patient is unable to see her/himself "realistically" as s/he is unable to distinguish between how her/his body appears and how it really is. Such a confused view about one's body is a result of an overly critical judgement of oneself. Women tend to be affected by BIDS to a much greater degree than men. Some medical researchers theorise that women adopt very strict body standards because of their low self-esteem; if women felt better about themselves, they would be less likely to overestimate their size (Bordo, 1993). Many social scientists link women's body dissatisfaction with the narrowly defined, unobtainable, unnatural body ideal instead of women's low self-esteem (Bordo, 1993; Peters, 1995). They argue that women's body dissatisfaction and low self-esteem are not causing eating problems. Rather, all these conditions are results of the unrealistic media ideal. While impossible to achieve, we are bombarded with messages stressing the desirability of such looks. Confronted by such an ideal, it is no wonder that women feel dissatisfied with their bodies, troubled with food and pressed to exercise at high intensity levels.

The process of bodily transformation requires hard work. The body has to be disciplined if we aim to attain the desired results. Foucault (1979) theorises that through such bodily discipline we accept a body ideal created by someone else. Consequently, the bodywork can become a tool of dominance. This means that when this ideal dominates our thoughts, exercise and diet effectively serve as disciplinary practices to restore certain order in society. Through discourses like consumerism, we are persuaded that it is necessary to spend time reshaping our bodies instead of worrying about inequality, hunger or environmental pollution. While engaged in body work, we have no time to think about what really matters 
in life. As we assume that body regimes are necessary, we also start believing that other ideas pushed by the dominant powers are essential. The beauty of this arrangement, Foucault continues, is that we inflict the discipline on ourselves through surveying the defects on our bodies. We watchfully aim to maintain our bodies within the accepted ideal without really pondering why such an ideal is desirable or why we spend so much time on body shaping.

Featherstone (1991) adds that as the consumption of body maintenance goods increases, the required time for body care and maintenance also increases. In this process, the body is treated instrumentally like any other consumer object. It is no wonder then that machine metaphors are often used to illustrate the importance of body maintenance. Like cars, Featherstone explains, "bodies require servicing, regular care and attention to preserve maximum efficiency" (p. 182). Interestingly, Linda Donalson (1996/1997) in NZF discusses body maintenance with this exact analogy. In her article, she contends that "Our bodies, like cars, need regular care to keep them in top condition" (p. 33) and provides maintenance tips for different body parts. Her article also illustrates that, like machines, bodies can be dissembled piece by piece for a closer scrutiny of possible defects.

Naturally, keeping the body properly tuned requires knowledge about its structure. The body has become an object of scientific methods that treat it as a "special" biological machine (Rintala, 1995). Based on these studies, we know how to get the best results out of our machine. Physical training regimes are grounded on laboratory testing of human objects and bodywork, thus, derives it principles from scientific data. However, unlike machines, human's cannot repeat movements endlessly without negative effects like injuries, neither are all humans created the same. Many humans tend to get bored with scientifically efficient training programmes and drop out as a result (Rintala, 1995). In addition, when we train our bodies like machines we loose touch of our feelings and emotions about our bodies. We are no longer humans, we have become robotic machines. The analogy of the body as a machine that requires regular servicing also implies that the body has become an object for us. Like other commodities within consumer culture, the body is treated as "plastic" (White, Young \& Gillett, 1995). We believe that the body form can be changed when we so desire. Therefore, within consumer culture the relationship between the body and self has developed to further emphasise the superiority, domination and control of the mind over the body.

When we vision our bodies as malleable, we also consider them as something we control. Body shape, like health, has become an individual's responsibility and a healthy looking body is a visible indicator of one's ability to fulfil this responsibility (White, Young \& Gillett, 1995). Conversely, individuals deviating from the ideal shape, like obese people, are commonly subjected to moral judgements. Obesity is connected with gluttony, laziness and sloth, because these people have failed to properly discipline themselves (Arveda Kissling, 1991). If they just ate less and exercised more, their bodies would transform into the ideal thinness. They are simply avoiding responsibility for their bodies and responsibility for the increased public spending on health care. Through such conceptualisation, the body becomes an object subservient to our 
(minds') orders. Body becomes a machine and like other technology within consumes culture, it needs fixing, tuning and repair work.

\section{CONCLUSION}

Evidently, the relationship between health and physical activity is complex as both concepts assume different meanings in different contexts. In the name of health, professional texts focus on the potential of physical activity for illness prevention, yet the popular media attaches physical activity to improved body shape. Such meanings are socially constructed to reflect the aspirations of the people who present us with the fitness information. As I demonstrated, both of these approaches are problematic. Although each approach defines health in a different manner, both seem to push the responsibility of health maintenance to the individual. Sedentary people are blamed for an unwillingness to take on an exercise programme or engage in bodily transformation. They become a burden to the public health care system. Or they begin to signify sloths as they have failed to build a "healthy" body. Despite such moral judgements, numbers of physically active people remain low.

In part, high inactivity levels might be a result of research techniques measuring people's engagement in physical activity. For example, questionnaires mapping physical activity levels have adopted ASCM definitions and most people do not find themselves participating in that type of exercise. The latest physical activity survey in New Zealand (Ministry of Health, 1992) exemplifies the possibility of a skewed view of activity levels as it was interested only in respondents' vigorous activity. The survey defined vigorous as "anything that made you breathe hard or sweat" (p. 44). Therefore, many people walking regularly would have been coded as inactive. However, it is unlikely that limitations in research techniques are the only explanation for people's inactivity. Perhaps, it is not the activity itself that is unappealing, but the way that it is promoted. Obviously, moral judgements have failed to inspire people to become physically active, but what alternatives do we have?

Undoubtedly, good physical condition helps to prevent illness, but as demonstrated earlier, a single emphasis on the role of physical activity in disease control can become problematic. To minimise such problems as individualism and victim blaming, we could place less emphasis on illness prevention and focus more on such positive aspects as fun, opportunity to socialise and convenience of physical movement. Perhaps is it less crucial to calculate the exact amount and intensity of such activity than to tune into its playfulness. Often physical activity is performed together with a group of people (a game of touch rugby or group exercise) and many participants derive enjoyment from such social contacts. We do not always need to reserve special time for "exercise." For some participants it is important to incorporate physical activity within everyday tasks. Following the principles of active living, walking to shops instead of driving, taking stairs instead of the elevator or taking care of the lawn with manual instead of motorised lawn mower can comprise daily physical activity. Therefore, happiness might prolong life more than a well planned, but rigid, laborious and boring exercise regimen. Physical activity that is convenient, fun and social might 
motivate more people to participate than physical activity that is scientifically designed to control disease.

In addition to fun, physical activity can be functional. Therefore, the emphasis of exercise advise should shift from body form to functionality. Although regular exercise might result in bodily transformation, the ideal that we aspire to emulate is impossible and non functional for most people. Few people need to be extremely thin or extremely muscular in today's society. Therefore, to avoid the problems deriving from extreme body consciousness, physical activity could emphasise functionality. For example, many people have weak trunk stabiliser muscles (external and internal obliques, iliopsoas and erector spinae) and as a result of such weaknesses they suffer from postural misalignments that can cause severe pain. Therefore, resistance training should improve the functionality of these body parts instead of increasing leg tone or biceps size. Conversely, some muscle groups (such as trapezius, gastrocnemius or quadriceps) can become tight due to constant tension in every day life. These muscle groups need relaxation or stretching exercises to retain their functionality. Similarly, we should detect the purpose of any physical activity and prioritise the functional practices. Although such practical, physical advice seems unrelated to the social construction of meaning, we need to alert ourselves, our students and our customers how fitness practices evolve from the social discourse.

Health, physical fitness, exercise, physical activity and active living are socially defined concepts. Making sense of their meanings and the resulting fitness practices requires an investigation into the premise of each concept. Such awareness helps us to select the type of physical activity we prefer and be informed consumers of the ever growing fitness information. Hopefully, then instead of guilt we promote the enjoyment of physical activity. Hopefully, instead of judgement we promote the education of healthy physicality.

\section{REFERENCES}

Arveda Kissling, E. (1991). One size does not fit all, or how I learned to stop dieting and love the body. QUEST, $42,351-147$.

Bartley, M. (1985). Coronary health disease and the public health 1850-1983. Sociology of Health and Illness, 7, 289-313.

Bartky, S. (1988). Foucault, femininity, and the modernization of patriarchal power. In I. Diamond, \& L. Quinby (Eds.), Feminism \& Foucault: Reflections on resistance (pp. 61-86). Boston: Northeastern University Press.

Blair, S. N., Brill, P. A., \& Barlow, C. E. (1994). Physical activity and disease prevention. In H. A. Quinney, L. Gauvin, \& A. E. T. Wall (Eds.), Toward active living (pp. 25-31). Champaign, IL: Human Kinetics.

Bordo, S. (1993). Unbearable weight: Feminism, western culture, and the body. Berkley and Los Angeles: University of California Press.

Bouchard, C. (1994). Active living from the biological science perspective: A word of caution. In H. A. Quinney, L. Gauvin, \& A. E. T. Wall (Eds.), Toward active living (pp. 277-279). Champaign, IL: Human Kinetics.

Bray, A. (1994). The edible woman. Media Information Australia, 72, 4-10.

Chernin, K. (1981). The obsession: Reflections on the tyranny of slenderness. New York: Harper \& Row. 
Crawford, R. (1980). Healthism and the medicalization of everyday life. International Journal of Health Services, 10 (3), 365-388.

Dishman, R. K. (1982). Compliance/adherence in health-related exercise. Health Psychology, 1, 237-267.

Donalson, L. (1996/1997, December/January). Warrant of fitness. New Zealand Fitness, 22, 32-35.

Edwards, P. (1994). Active living: A critical examination. In H. A. Quinney, L. Gauvin, \& A. E. T. Wall (Eds.), Toward active living (pp. 285-287). Champaign, IL: Human Kinetics.

Featherstone, M. (1991). The body in consumer culture. In M. Featherstone, M. Hepworth \& B., S. Turner (Eds.), The Body: Social processes and cultural theory (pp. 170-196). London: Sage.

Frank, A. W. (1991). For a sociology of the body: An analytical review. In M. Featherstone, M. Hepworth, \& B., S. Turner (Eds.), The Body: Social processes and cultural theory (pp. 36-102). London: Sage.

Foucault, M. (1979). Discipline and Punish. New York: Vintage.

Haskell, W. L. (1995). More vs less. IDEA Today, 13 (9), 40-44.

Haskell, W. L. (1994). Physical/physiological/biological outcomes of physical activity. In H. A. Quinney, L. Gauvin, \& A. E. T. Wall (Eds.), Toward active living

(pp. 17-23). Champaign, IL: Human Kinetics.

Homlund, C. A. (1989). Visible difference and flex appeal. Cinema Journal, 28 (4), 38-51.

Ingham, A. (1985). From public issue to personal trouble: well-being and the fiscal crisis of the state. Sociology of Sport Journal, 2, 43-55.

Kravitz, L., \& Robergs, R. (1993). To be active of not to be active. IDEA Today, 11 (3), 47-53.

Malina, R. M. (1994). Benefits of physical activity from a lifetime perspective. In $\mathrm{H}$. A. Quinney, L. Gauvin, \& A. E. T. Wall (Eds.), Toward active living (pp. 47-53). Champaign, IL: Human Kinetics.

Markula, P. (1995). Firm but shapely, fit but sexy, strong but thin: The postmodern aerobicizing female bodies. Sociology of Sport Journal, 12, 424-452.

Martin, L. H, Gutman, H., \& Hutton, P. H. (1988). Technologies of the Self: A seminar with Michel Foucault. Armherst: University of Massachusetts Press.

New Zealand Health Information Service (1995). Shaping Up: A profile of New Zealanders' patterns of smoking, drinking, exercise and body mass index 1992/93. Ministry of Health: Health Research and Analytical Services.

Paffenberger, R. S., Hyde, R. T., Wing, A. L., Lee, I-M., \& Kambert, J. B. (1994). An active and fit way-of-life influencing health and longevity. In $\mathrm{H}$. A. Quinney, L. Gauvin, \& A. E. T. Wall (Eds.), Toward active living (pp. 61- 68). Champaign, IL: Human Kinetics.

Peters, N. (1995). The ascetic anorexic. Social Analysis, 37, 44-66.

Pollock, M. L., Feigenbaum, M. S., \& Brechue, W. F. (1995). Exercise prescription for physical fitness. QUEST, 47, 320-337.

A report of the Surgeon General (1996). Physical activity and health. U.S Department of Health and Human Services.

Rintala, J. (1995). Sport and technology: Human questions in a world of machines. Journal of Sport $\mathcal{E}$ Social Issues, February, 62-75. 
Shephard, R. J. (1995). Physical activity, fitness, and health: The current consensus. QUEST, 47, 288-303.

Sparkes, A. C. (1991). Alternative visions of health-related fitness: An exploration of problem-setting and its consequences. In N. Armstrong \& A. Sparkes (Eds.), Issues in physical education (pp. 204-277). London: Cassell Educational.

Spitzack, C. (1990). Confessing excess: Women and the politics of body reduction. Albany, NY: State University of New York Press.

White, P. G., \& Gillett, J. (1994). Reading the muscular body: A critical decoding of advertisements in Flex magazine. Sociology of Sport Journal, 11, 18-39.

White, P., Young, K., \& Gillett, J. (1995). Bodyworks as a moral imperative: some critical notes on health and fitness. Society and Leisure, 18 (1), 159-182.

Wolf, N. (1990). The beauty myth. London: Vintage. 